\title{
ANÁLISIS ESTRUCTURALISTA DE LA TEORÍA DE LOS GRUPOS DE REFERENCIA*
}

\author{
Cláudio Abreu \\ Universidade de São Paulo \\ bttp://dx.doi.org/10.15304/ag.39.1.5679
}

\section{Resumen}

El objetivo de este artículo es presentar una reconstrucción, conforme a la metateoría estructuralista, de la teoría de los grupos de referencia. La teoría, desarrollada por Robert Merton, ha sido largamente utilizada en los más variados campos de las ciencias sociales, aunque con escasa consideración metateórica hasta ahora. La reconstrucción atenderá a la presentación que de la misma se recoge en sendos artículos de su libro Social Theory and Social Structure, a saber: "Contributions to the theory of reference group behavior" y "Continuities in the theory of reference groups and social structure". Después de una consideración informal de la teoría, serán presentados sus modelos potenciales, modelos, modelos potenciales parciales, condiciones de ligadura, vínculos interteóricos y aplicaciones intencionales.

Palabras clave: filosofía de las ciencias sociales, Robert Merton, metateoría estructuralista.

\begin{abstract}
The goal of this article is to present a reconstruction, according to the structuralist metatheory, of the reference groups theory. The theory developed by Robert Merton is a theory largely used in the most varied fields of social sciences but, still up to this day, it does not have a more accurate treatment from the metascientific standpoint. The reconstruction will attend the presentation of it is reflected in two articles in his book Social Theory and Social Structure, namely: "Contributions to the theory of reference group behavior" and "Conti-
\end{abstract}

Recibido: 16/12/2018. Aceptado: 24/01/2019.

" Este trabajo fue financiado por el proyecto de investigación PICT-2014-1741 de la Agencia Nacional de Promoción Científica y Tecnológica (ANPCyT, Argentina). Agradezco los valiosos comentarios de los evaluadores anónimos. 
nuities in the theory of reference groups and social structure". After an informal account of the theory will be presented their potential models, models, partial potential models, constraints, intertheoretical links and applications intended.

Keywords: philosophy of social sciences, Robert Merton, structuralist metatheory.

\section{Introducción}

La teoría de los grupos de referencia (TGR) es largamente utilizada en los más variados campos de las ciencias sociales, pero hasta ahora no tuvo un tratamiento preciso desde el punto de vista metacientífico. Aquí se presenta TGR tal y como ha sido presentada por Merton en sus célebres artículos integrados en Social Theory and Social Structure, a saber: "Contributions to the Theory of Reference Group Behavior" y "Continuities in the Theory of Reference Groups and Social Structure". ${ }^{1}$

El objetivo de este artículo es presentar una reconstrucción de la teoría de los grupos de referencia. Para presentar esta reconstrucción me apoyaré en el instrumental de la Metateoría Estructuralista. Después de una consideración informal de la teoría, serán presentados sus modelos potenciales, modelos, modelos potenciales parciales, condiciones de ligadura, vínculos interteóricos y aplicaciones intencionales.

Conviene señalar que, a los efectos de la reconstrucción en el marco estructuralista desde una perspectiva sincrónica, TGR se concibe como un elemento teórico aislado, es decir, no formando parte de ninguna red teóri$c a$. Ello se debe a que los aportes de Merton no hacen suficiente referencia ${ }^{2}$ a posibles distinciones relevantes que pongan en escena la eventualidad de que TGR tenga algunas especializaciones. ${ }^{3}$ Posibles desarrollos de ellos, en

${ }^{1}$ Los dos capítulos aparecen en la tercera edición del libro (1968). La primera edición es de 1949.

2 Hay alguna referencia hecha por Merton a situaciones en que el individuo en determinada circunstancia tenga más de un grupo de referencia. Eso podría, quizás, dar pie a pensar en especializaciones o, otra posibilidad, en otro constructo teórico muy emparentado a TGR. Con más de un grupo involucrado en la situación, el comportamiento del individuo estaría dependiente también de un criterio que posibilite al individuo decidir cual de los grupos tiene mayor "fuerza" en la situación. Eso llevaría a que la estructura conceptual de tal constructo fuera en algo distinta de la de TGR. Sin embargo, la presente observación, no más que puramente intuitiva, necesita ser confrontada con un estudio sistemático del desarrollo de la teoría.

${ }^{3}$ Las teorías suelen presentar diversos niveles de especificidad: partiendo de un nivel muy general, paso a paso se va concretando en direcciones diversas, siendo estas direcciones cada vez más restrictivas y específicas. Este paso de un nivel a otro más restricto y específico es lo que constituye la relación de especialización. 
la línea de desplegar diferentes especializaciones para diferentes grupos de aplicaciones intencionales, convertirían lo aquí presentado en un elemento teórico básico de una red teórica.

Finalmente, importa decir, este análisis de TGR, juntos con otras contribuciones semejantes - Lorenzano y Abreu (2010), Abreu (2012 y 2014), Sota (2013), por ejemplo-, demuestra ante todo que que la Metateoría Estructuralista, mayormente conocida por sus aportes a las ciencias naturales, es también aplicable a las ciencias sociales. Estos pequeños pasos dados en esta dirección hacen plausible postular que, como ocurre en el caso de las ciencias naturales, la Metateoría Estructuralista puede contribuir significativamente a los debates llevados a cabo en la filosofía de la sociología. Ahora bien, para discutir temas como leyes, contrastación y otros para el caso de las teorías sociológicas importa tener a mano reconstrucciones como la que este trabajo presenta. Por otra parte, en lo que respecta más específicamente a la sociología, acercarse a la discusión acerca de la distinción sociología teórica/sociología empírica es plenamente posible tenendo a mano nada más que la concepción de teoría que brinda la Metateoría estructuralista. Ciertamente hay mucho que aportar desde un nivel general, en este sentido; pero un acercamiento a una cuestión de ese tipo con la posibilade de trabajarse casos concretos, ciertamente seria mucho más productivo.

\section{Aclaraciones preliminares}

Parece oportuno empezar por aclarar que, según Merton, es característico de las teorías de alcance intermedio que dichas teorías "no permanecen separadas, sino que se reúnen en redes más amplias de teorías, como se ilustra con las teorías del nivel de aspiración, grupo de referencia y estructura de oportunidad" (Merton 1968, p. 68 [2002, p. 97]), lo que abonaría la idea de que TGR formaría parte de una unidad teórica superior, ya sea como especialización (al igual que lo serían las teorías del nivel de aspiración y de la estructura de oportunidad) de una red teórica (por construirse) o como componente incrustado de algún modo a otra teoría (red teórica) más amplia (que también contemple la incrustación, ${ }^{4}$ de algún modo, de las teorías del nivel de aspiración y de la estructura de oportunidad).

\footnotetext{
${ }^{4}$ Intuitivamente, la incrustación acontece cuando las situaciones a las que una teoria pretende dar cuenta, incluyento no sólo los casos de logro, pero también aquellos en que dicha teoría falla en su pretensión, son incorporadas por otra teoría, que logra dar cuenta de todos ellos.
} 
$\mathrm{Al}$ respecto, tal y como asume explícitamente Merton al principio del segundo de los artículos referentes a TGR, "Continuities in the Theory of Reference Groups and Social Structure”, “[sus] páginas [...] están organizadas en relación con problemas teóricos, tanto de grupos de referencia como de materias afines a la estructura social en general" (Merton 1968, p. 335 [2002, p. 362]). Este comentario nos ayuda a entender una afirmación de Merton al final del mismo artículo, cuando escribe acerca de las consecuencias de la conducta relativa a grupos de referencia que:

Al poner fin a esta exposición sobre continuidades de la teoría de la conducta relativa a grupos de referencia, me limito a mencionar, más bien que a analizar, problemas escogidos de las consecuencias de diferentes tipos de conducta relativa a grupo de referencia. Estudiarlos con el detalle que ahora es posible sería convertir esto en todo un libro extenso más bien que en una información provisional. (Merton 1968, p. 438 [2002, p. 468])

Con independencia de la extensión, parece que las consecuencias a las que se refiere no requieren necesariamente ser consideradas para identificar lo que es central para TGR. Además, al tratar específicamente de las funciones y disfunciones de la conducta relativa a un grupo de referencia, dice:

[...] hay 'coherencia entre la teoría de grupo de referencia y ciertos conceptos de la sociología funcional". Parece que estos se refieren a aspectos diferentes del mismo asunto: uno se enfoca sobre el proceso mediante el cual los individuos se relacionan con los grupos y refieren su conducta a los valores de los grupos; los otros se enfocan sobre las consecuencias de los procesos primordialmente para las estructuras sociales, pero también para los individuos y los grupos comprendidos en las estructuras sociales'. (Merton 1968, p. 438 [2002, p. 468])

Así tenemos que Merton afirma que TGR, dicho de modo general, "trata de sistematizar los determinantes y consecuencias de los procesos de valorar y de auto-estimación en que los individuos toman los valores o las normas de otros individuos como sistema comparativo de referencia" (Merton 1968, p. 228 [2002, p. 314]). Pero además, presenta ideas como la de que "la función de la conformidad es la aceptación por el grupo, así como la progresiva aceptación por el grupo refuerza la tendencia a la conformidad" (Merton 1968, p. 308 [2002, pp. 334-335]). Aquí, con la reconstrucción que proponemos, tratamos de elucidar lo que concierne a la explicación de la conducta relativa a grupos de referencia. En tanto que entendemos que las cuestiones relativas a 'determinantes', 'consecuencias' y 'función', a las que se hace referencia en las anteriores citas, aunque tienen que ver indirectamente con TGR, no constituyen lo que dicha teoría es en sí misma.

Sin embargo, esta mezcla de problemas y/o perspectivas teóricas presentes en dichos artículos parece ser una muestra paradigmática de lo que 
Merton expresa por medio de la noción de 'alcance intermedio', no tanto por lo que se refiere a las teorías de alcance intermedio, sino más bien a la orientación de alcance intermedio, o sea, a la idea de que:

La teoría sociológica, si ha de avanzar de manera significativa, debe proceder en estos planos interrelacionados: 1) desarrollando teorías especiales sobre las cuales derivar hipótesis que se puedan investigar empíricamente y 2) desarrollando, no revelando súbitamente, un esquema conceptual progresivamente más general que sea adecuado para consolidar grupos de teorías especiales. (Merton 1968, p. 51 [2002, p. 68])

Esta contextualización hace pensar que Merton entiende su estudio de los grupos de referencia como una oportunidad de sofisticación del funcionalismo. La noción de 'función' (estrechamente vinculada con la noción de 'consecuencias') permite retomar aspectos relevantes para Merton, como, por ejemplo, el de la conformidad o la inconformidad con el intra-grupo. Con ello se estaría apuntando a un vínculo importante con la teoría de la anomia. Lo que hay entonces son relaciones entre 'teorías especiales' en un proceso de 'revelación de un esquema conceptual progresivamente más general'.

\section{Teoría de los grupos de referencia}

Así pues, TGR pretende explicar, en ciertas circunstancias, el comportamiento del individuo en lo que concierne a su actitud frente a los valores de un colectivo (que presenta determinados valores como característicos, es decir, un colectivo normativizado), y lo hace vinculando, por medio de la noción de referencia, la actitud de un individuo con su aspiración a respecto de afiliarse-a/integrarse-en ${ }^{5}$ este colectivo.

El concepto de grupo de referencia se originó en la psicología social; sin embargo, Merton defiende que "el concepto de grupo de referencia tiene también un lugar distintivo en la teoría de la sociología, con su enfoque sobre la estructura y funciones de los ambientes sociales en que están situados los individuos" (Merton 1968, p. 335 [2002, p. 362]). En todo caso, es importante aclarar que "la expresión ahora consagrada de 'grupo de referencia' tiene algo de inapropiado, porque se aplica no sólo a grupos, sino también a individuos y a categorías sociales" (Merton 1968, p. 338 [2002, p. 365]).

${ }^{5}$ Tanto para el caso de "asimilar/adaptarse-a" como para el de "afiliarse-a/integrarse-en" los pares de expresiones pueden considerarse sinónimas, siendo más apropiado un miembro del par que otro según el contexto de uso. A fin de evitar lo farragoso que sería mantener los pares de expresiones a lo largo del texto, a partir de aquí privilegiaré el uso de "asimilar" y de "afiliarse a" (prescindiendo en este caso del guion del que hice uso previamente). 
Un individuo de referencia es aquel que tiene cierto status social diferenciado, como puede ser un artista renombrado, un importante deportista etc. Reconociendo que, por una cuestión de simplicidad, en la tradición sociológica se utiliza en general la expresión 'grupo de referencia' para designar también individuos de referencia, Merton procura aclarar esta última noción, y lo hace caracterizándola a partir de sus semejanzas y diferencias con respecto a la noción de papeles sociales.

El individuo de referencia ha sido descrito con frecuencia como un modelo del papel. Pero, como implican las palabras mismas, el supuesto de que sean cosas conceptualmente sinónimas oscurece una diferencia fundamental en las materias de las cuales se refieren respectivamente. La persona que se identifica con un individuo de referencia tratará de aproximarse a la conducta y valores de aquel individuo en sus diferentes papeles sociales. El concepto de modelo de papel puede tomarse en un sentido más restringido que denota una identificación más limitada con un individuo sólo en uno o en unos pocos papeles seleccionados. (Merton 1968, pp. 656-357 [2002, p. 384])

En lo que concierne a los grupos "se entiende en general que el concepto sociológico de grupo se refiere a un número de personas que actúan entre sí de acuerdo con normas establecidas" (Merton 1968, p. 339 [2002, p. 366]). Las relaciones sociales consagradas y características (formas normadas de interacción) de cada grupo acaban por caracterizarse como aspectos identificables de la estructura social.

A su vez, las categorías sociales son caracterizadas por Merton como "agregado de situaciones sociales cuyos ocupantes no están en interacción social. Tienen características sociales idénticas — de sexo, edad, situación matrimonial, ingreso etc-, pero no están orientadas necesariamente hacia un cuerpo de normas distintivo y común" (Merton 1968, p. 353 [2002, p. 381]).

Es importante en este contexto tener presente que hay que establecerse una relación entre un individuo y un grupo (que puede estar dado por un único individuo, como ya se señaló) para que éste pueda servir como referencia de aquél. Esto queda más claro si percibimos "que el concepto de la conducta relativa a grupo de referencia presupone algún conocimiento o imagen de las normas y los valores predominantes en el grupo" (Merton 1968, p. 391 [2002, p. 419]). Para concretar puede decirse que TGR "se enfoca sobre el proceso mediante el cual los individuos se relacionan con los grupos y refieren su conducta a los valores de los grupos" (Merton 1968, p. 280 [2002, p. 306]). Por 'valores del grupo' se debe entender cosas como 'normas', 'cánones', 'patrones', 'hábitos', 'prácticas', 'rutinas', 'usos', 'modas', 'costumbres', 'tradiciones', 'etiqueta', 'protocolos', 'ritos', 
'procedimientos', 'reglas', etc. La referencia a estos valores puede ser: (i) positiva, cuando "comprende la asimilación motivada de las normas del grupo o de los patrones del grupo" (Merton 1968, p. 354 [2002, p. 382]); o (ii) negativa, cuando existe "el rechazo motivado, es decir, no sólo la mera no aceptación de las normas, sino la formación de contranormas" (Merton 1968 , p. 354 [2002, p. 382]); o (iii) neutra, cuando los individuos son completamente indiferentes ante la perspectiva de pertenecer o no pertenecer al grupo - esta clase de individuos consta de los que no se orientan en absoluto hacia el grupo ni positiva ni negativamente, pero que podrían hacerlo, pues tienen cierta interacción con (conocen) el grupo en cuestión. ${ }^{6}$

Los comentarios previos parecen dar a entender que los grupos de referencia son sin más determinados colectivos o grupos de individuos; es decir, lo que podríamos representar como ciertos conjuntos de individuos (incluidos algunos conjuntos unitarios). Sin embargo, considerarlos así conlleva perder un rasgo decisivo de los grupos de referencia, a saber, que un determinado colectivo o grupo de individuos pasa a ser grupo de referencia en tanto que lo es para un determinado individuo en determinada circunstancia. No hay grupos de referencia en términos absolutos, los hay relativamente a un individuo y una circunstancia. Que ser grupo de referencia es relativo a un individuo debe resultar intuitivamente obvio, toda vez que ser grupo de referencia parece que requiere la adopción de una actitud, un posicionamiento determinado, por parte de ese individuo. Que sea relativo a una circunstancia parece que es apropiado por un par de razones. En primer lugar, lo que sea un grupo de referencia para un individuo en un determinado momento no tiene porqué serlo en otro momento. Esta mera razón daría lugar a que en vez de "circunstancias" se pensara en que la noción de "grupo de referencia" estuviera relativizada a "momentos" (además de estarlo a "individuos"), pero lo cierto es que en el mismo momento un mismo individuo podría considerar dos o más colectivos como grupos de referen-

${ }^{6}$ La noción de referencia neutra sirve para capturar un fenómeno que es distinto, por ejemplo, del caso en el que el individuo no pertenece a un grupo y es indiferente a pertenecer o no a él porque no está en la órbita de este grupo; o sea, ni siquiera mantiene algún tipo de relación con él. La noción de referencia neutral captura el fenómeno donde un individuo mantiene relación con un grupo (sea como sea esta relación) y, sin embargo, este grupo no sirve de (no está como) guía para este individuo: no se da el caso de ser ni referencia positiva ni referencia negativa para este individuo. Estrictamente no se da la referencia, pero eso no le quita (a la noción de referencia neutral) su valor (y utilidad). Es común la utilización de una noción similar en las encuestas (sea para publicidad, actividades políticas u otras) donde se procura identificar, por ejemplo, la aceptación y rechazo de cierto producto o de cierta persona por parte de la población. 
cia para él (por ejemplo, para un chico dado, una pandilla del barrio, por un lado, y un grupo de compañeros en el centro de estudios, por otro lado). Por eso, en segundo lugar, debe considerarse una noción que intuitivamente sea más compleja que la de "momentos temporales" (que capture la idea de contexto estructural), llamémosla "circunstancia”, respecto a las cuales se fija qué colectivos son grupos de referencia; aunque a los efectos de este trabajo las presentaremos como entidades simples. Preservaremos, pues, la noción de grupo de referencia como una noción dependiente de otras tres más básicas; a saber: "individuos", "colectivos"7 y "circunstancias".

Teniendo esto en cuenta, podemos decir que la hipótesis central de la teoría, en su versión positiva (es decir, de referencia positiva) es que “[...] en la medida en que los individuos de un grupo subalterno o en perspectiva se sienten movidos a afiliarse a un grupo, tenderán a asimilarse a los sentimientos y adaptarse a los valores del estrato del grupo que tiene autoridad y prestigio" (Merton 1968, p. 308 [2002, pp. 334-335]). En base a esto, Merton afirma que en casos como los de

[u]n soldado raso del ejército que busca el ascenso, sólo en un sentido estrecho y teóricamente superficial puede considerarse que siga una conducta diferente de la de un inmigrante que asimila los valores de un grupo nativo, o de un individuo de clase baja que se ajusta a su concepto de las normas de conducta de la clase media alta, o de un muchacho en una zona de barrios bajos que se orienta hacia los valores de un trabajador de casa de beneficencia y no hacia los valores de la pandilla de la esquina, o de un estudiante de Bennington que abandona las ideas conservadoras de sus padres para adoptar las ideas más liberales de sus compañeros de colegio universitario, o de un católico de clase humilde que se aparta de la norma de su intra-grupo votando por los republicanos, o de un aristócrata francés del siglo XVIII que se alinea en un grupo revolucionario de la época. (Merton 1968, p. 332 [2002. pp. 359-360])

Todos ellos son casos que responden a la pauta dada por TGR.

Parece claro que la conducta de un individuo, según nos propone Merton, se caracteriza por ser la expresión de un sentimiento por parte de ese individuo en relación al grupo. Según sea este sentimiento, el grupo será de referencia positiva, negativa o neutra. Puede ser el caso de una "asimilación motivada de las normas del grupo", "[d]el rechazo motivado" de dichas normas (Merton 1968, p. 354 [2002. p. 382]) o de la neutralidad; es decir, referencia positiva, negativa y neutra, respectivamente. En este sentido, aspirar a pertenecer al grupo, por ejemplo, supone aceptación de los valores del grupo, querer ser/seguir siendo del grupo es (presupone) aceptar lo que

7 Hasta aquí se habló de "colectivos" o "grupos". A partir de ahora se privilegiará la expresión de "colectivos" para evitar confusiones entre las expresiones de "grupo" y de "grupo de referencia". 
acepta el grupo. Aspirar a abandonar/a no pertenecer al grupo es (supone) rechazar lo que acepta el grupo. Finalmente, están aquellos que no se orientan en absoluto hacia el grupo en cuestión.

\section{El elemento teórico básico de TGR}

\subsection{La clase de los modelos potenciales de TGR}

En el caso de TGR entre los constituyentes de la teoría tenemos: "individuos", "colectivos", "valores", "circunstancias" y "evaluaciones". Éstos son los dominios a partir de los cuales se darán (conjuntistamente) las funciones "normativizar", "comportarse", "aspirar" y "ser grupo de referencia, para un individuo en una circunstancia". Teniendo en cuenta estos componentes conceptuales (y presuponiendo que el campo de acción del individuo está limitado por el conjunto de relaciones que tiene con los distintos colectivos existentes), ya es posible caracterizar el conjunto de los modelos potenciales de TGR.

D1: $x=\langle I, C, V, S, E$, nor, com, asp, gru $\rangle$ es un modelo potencial de la teoría de los grupos de referencia $\left(x \in \mathbf{M}_{\mathrm{p}}(\mathrm{TGR})\right)$ syss:

(1) $I \neq \varnothing \wedge\|I\|<\aleph_{0}$ ("individuos”)

(2) $C \subseteq(\operatorname{Pot}(I)-\varnothing)$ (“colectivos")

(3) $V \neq \varnothing \wedge\|V\|<\aleph_{0}$ ("valores")

(4) $S \neq \varnothing \wedge\|S\|<\aleph_{0}$ ("circunstancias")

(5) $E=\{+,-$, ? $\}$ ("evaluaciones")

(6) nor: $C \times S \rightarrow(\operatorname{Pot}(V)-\varnothing)$ ("normativizar")

(7) com: $I \times S \times \mathrm{D}_{\text {II }}($ nor $) \rightarrow E$ ("comportarse")

(8) asp: $I \times S \times C \rightarrow E$ ("aspirar")

(9) gru: $I \times S \rightarrow C$ ("ser grupo de referencia, para un individuo en una circunstancia")

Comentarios a este predicado:

(1) I representa el conjunto de "individuos": seres humanos.

(2) C representa el conjunto de "colectivos": desde un "colectivo unitario" formado por un solo individuo, pasando por grupos de personas con cierta identidad de grupo asumida por sus miembros (identidad que proporcionan unas normas, más o menos explícitas, compartidas), hasta, incluso, categorías sociales, determinadas por una característica, que comprenden fenómenos como, por ejemplo, los "individuos de un sexo" o las "clases sociales". Lo que es distintivo de estos 
colectivos, así caracterizados, es que tienen como condición necesaria el estar constituidos por individuos. Pueden ser grupos que existen actualmente o que no existan más (es decir, que hayan existido y desaparecido), pueden ser grupos cerrados (aquellos que no permiten o dificultan en mucho el ingreso de nuevos integrantes) o abiertos (aquellos que permiten el ingreso de nuevos integrantes, muchos incluso los buscan constantemente), pueden ser grupos a los cuales se pertenece o no, pueden ser grupos a los cuales ya se perteneció y hoy ya no se pertenece; un colectivo puede ser un grupo al que se aspire ingresar, del que resulte indiferente la afiliación, o incluso del que se tenga razones para desear no pertenecer. No toda posible combinación de individuos es uno de estos colectivos, sólo ciertas combinaciones lo son, es decir, solo ciertas combinaciones son identificadas como tal, son concebidas como una cosa del mundo social (por ejemplo, no sería un colectivo para nuestra teoría el resultante de considerar conjuntamente un individuo de Caracas, otro de Londres y otro de Praga que no tuvieran nada socialmente relevante que ver entre sí).

(3) V representa el conjunto de "valores": normas, cánones, patrones, hábitos, rutinas, usos, modas, costumbres, tradiciones, etiquetas, protocolos, ritos, procedimientos, prácticas, reglas,..., que están presentes en la sociedad constituyendo la moral de los grupos sociales. Los valores son principios que permiten orientar los comportamientos (o conductas) a adoptar; son creencias fundamentales que ayudan a preferir, evaluar y elegir unas cosas en lugar de otras, o unos comportamientos (o conductas) en lugar de otros; son normas que rigen las conductas.

(4) S representa el conjunto de "circunstancias": contextos estructurales. Captura el estado de cosas que sirve de escenario para que puedan "actuar" los individuos y los colectivos. Aunque podría ser caracterizada como una noción compleja (con estructura), no se hace así en esta reconstrucción por entenderse que la matización en nada contribuiría a un mejor entendimiento de la teoría en tanto que autónoma, tal y como se la considera aquí; es decir, siendo bastante clara la noción intuitiva de circunstancia, presentarla como una estructura no afectaría en nada lo que es central e importante para la reconstrucción a la luz de la consideración autónoma que aquí se hace de ella (otra cosa es que pueda tener interés esa consideración más estructurada de la circunstancias en un marco más amplio de teorías del que forme parte TGR). 
(5) E representa el conjunto de "evaluaciones": la positiva (+), la negativa (-) y la neutra (?). El uso de estos elementos en la reconstrucción es algo peculiar y, justamente por eso, será aclarado más adelante cuando aparezcan en las funciones correspondientes. En todo caso, como se verá de inmediato, son los valores que le corresponden al codominio de las funciones com y asp.

(6) nor representa la función "normativizar", que a un colectivo le asigna, en determinada circunstancia, un(os) elemento(s) del conjunto $V$. Esto es importante para TGR una vez que cierto conjunto de valores estará directamente involucrado (de manera positiva o negativa) o no estará involucrado (manera neutra) con el comportamiento de cierto individuo.

(7) com representa la función "comportarse", que a un individuo le asigna, en una determinada circunstancia y respecto de un conjunto de valores, un elemento del conjunto $E$. Los valores de com pueden ser, de acuerdo con el conjunto $E$, solo tres: +, -, ?. Si el valor es +, esto significa que el individuo en una determinada circunstancia se comporta de acuerdo con los valores. Si el valor es -, esto significa que el individuo en una determinada circunstancia se comporta en desacuerdo motivado con respecto a (en oposición a) los valores. Si el valor es ?, esto significa que el individuo en una determinada circunstancia se comporta de modo indiferente a los valores.

(8) asp representa la función "aspirar", que a un individuo, en una determinada circunstancia y respecto de un colectivo, le asigna un elemento del conjunto $E$. Los valores de asp pueden ser, de acuerdo con el conjunto $E$, solo tres: +, -, ?. Si el valor es +, esto significa que el individuo quiere (desea, aspira a) pertenecer al colectivo. Si el valor es -, esto significa que el individuo motivadamente no quiere (desea no pertenecer, aspira no pertenecer) al colectivo. Si el valor es ?, esto significa que el individuo es indiferente con respecto a pertenecer al colectivo.

(9) gru representa la función "ser grupo de referencia, para un individuo en una circunstancia". Asigna a un individuo, en cierta circunstancia, un colectivo que servirá de referencia para su comportamiento. ${ }^{8}$ Cuando el individuo asimila el conjunto de valores del colectivo, hay referencia positiva; cuando el individuo rechaza el conjunto de

${ }^{8}$ En otro trabajo (Abreu 2012) fueron presentadas algunas observaciones acerca del carácter relacional y disposicional de la noción de grupo de referencia. Estas características no serán tratadas en la presente reconstrucción. 
valores del colectivo, hay referencia negativa; cuando el individuo es indiferente, bajo las condiciones ya mencionadas, con respecto al conjunto de valores del colectivo, hay referencia neutra.

\subsection{La clase de los modelos de TGR}

D2: $x=\langle I, C, V, S, E$, nor, com, asp, gru $\rangle$ es un modelo de la teoría de los grupos de referencia $(x \in \mathbf{M}(\mathrm{TGR}))$ syss:

(1) $x \in \mathbf{M}_{\mathrm{p}}$ (TGR)

(2) para todo $i \in I, c \in C, v_{j(j \in \mathrm{N}, 1 \leq j \leq n)} \in V, s \in S, \beta \in E$ y siendo $\left\{v_{1}, \ldots, v_{k}\right\}$ $\in(\operatorname{Pot}(V)-\varnothing):$

$$
\operatorname{gru}(i, s)=c \longmapsto(\operatorname{asp}(i, s, c)=\beta \rightarrow \operatorname{com}(i, s, \operatorname{nor}(c, s))=\beta)
$$

Comentario sobre este predicado:

(1) Expresa la condición formal de que $x$, para ser modelo de TGR, pertenezca a los modelos potenciales de TGR.

(2) Expresa la ley fundamental de TGR, la que afirma que el individuo, en dada circunstancia, tiene un colectivo como referencia únicamente si fuera el caso que si tiene alguna aspiración con referencia a dicho grupo entonces su conducta en lo que se refiere a los valores de dicho colectivo estará de acuerdo con tal aspiración. Notese que $\beta$ debe ter un dos tres valores que pertenecen a $E$, y que tras el bicondicional se requiere que si el valor de asp es $\beta$, el valor de com sea el mismo.

\subsection{La clase de los modelos parciales de TGR}

Se pueden examinar los conceptos de TGR, a saber, $I, C, V, S, E$, nor, com, asp, gru, a fin de determinar cuáles de estos conceptos son TGRteóricos y cuáles son TGR-no-teóricos, tomando en cuenta que los TGRteóricos presuponen el principio o ley de TGR para la determinación de sus valores.

Comencemos con I ("individuos"). Considérese la caracterización informal de este concepto en los modelos potenciales de TGR. Así pues, I constituye uno de los conjuntos base principales que establecen la ontología empírica de la teoría. Sin embargo, para averiguar si una entidad particular pertenece al conjunto $I$ no es necesario presuponer la validez de la ley de TGR; bastarían, de hecho, métodos empíricos independientes, aquéllos que utilizamos para identificar observacionalmente los seres humanos y distinguirlos de las demás cosas existentes en nuestra vida cotidiana. 
Algo similar ocurre con C ("colectivos"). Este concepto es también un concepto que constituye otro de los conjuntos base principales que establecen la ontología empírica de la teoría; sin embargo, se trata de un conjunto derivado de $I$. A pesar de ese carácter derivado y dado su papel especialmente relevante en la teoría, se habla de la entidad que designa como "conjunto (o dominio) básico" forzando dicha noción. Que un conjunto de individuos cualquiera pertenezca a $C$ se determina empíricamente, con independencia de la ley fundamental de TGR. La observación del comportamiento y/o de algunas de las características de los individuos nos permite determinar las entidades que pertenecen a dicho conjunto. Con cada sujeto humano podemos conformar un colectivo, dado extensionalmente por el conjunto unitario al que ese sujeto pertenece. Un grupo es identificado como tal por una semejanza en las actitudes, las acciones, los comportamientos de sus integrantes; en algunos casos, semejanzas geográficas, educacionales, tecnológicas, pero todas ellas con la característica de que hay interacción entre los individuos. La determinación de sus integrantes puede darse por una sencilla observación (en muchos y variados casos) o por una investigación histórica por relatos orales o bibliográficos que relaten las actitudes, las acciones, los comportamientos de sus integrantes (grupos que no existen más). En grupos formales, como asociaciones, es posible buscar en los registros de la asociación el listado de integrantes, o sea, los documentos de la asociación, la carpeta para tomar lista en una clase, el anuncio con los listados de profesores del departamento de determinada asignatura y muchos otros documentos posibilitan la determinación. Por fin, en cuanto a las categorías sociales, la semejanza puede ser económica, educacional, tecnológica, etc., pero no necesariamente con interacción entre los individuos. La determinación de las entidades que componen este tipo de colectivo, una vez que se tenga establecido el criterio (digamos, varones entre 50 y 70 años de determinada ciudad), se establece, por ejemplo, por medio de encuestas, de acceso a bancos de datos que contengan dicha información, por el control de los registros de partidas de nacimiento (es una posibilidad, aunque en la práctica sea inviable, pues además se requiere el control de defunciones).

También $V$ ("valores"), que constituye otro de los conjuntos base principales que establecen la ontología empírica de la teoría, comprende los valores determinados empíricamente, con independencia de TGR. La determinación de las entidades que caen bajo este concepto puede darse por el acceso a estatutos, códigos de leyes (normas y reglas), por publicaciones especializadas (moda), por manuales (etiqueta, protocolo, rito, procedimiento), etc. 
En lo que se refiere a $S$ ("circunstancias"), el conjunto de contextos estructurales o circunstancias, parece darse lo mismo. Considerando la caracterización informal de este concepto al explicitar los modelos potenciales de TGR, debe recordarse que ésta es una noción intuitiva idealizada. De hecho es una noción vaga que podría comprender, además del componente temporal, otros factores respecto de los cuales relativizar, por ejemplo, la combinación de valores asumida por un colectivo o el comportamiento de un colectivo. Entre esos factores encontraríamos eventos (que incluyen el componente temporal) o aspectos geográficos junto a un componente temporal (considérese cómo los valores asumidos por un grupo pueden, en determinado momento, estar condicionados por eventos como un estallido social o por estar viviendo en una determinada zona). La determinación de las circunstancias para cada aplicación de la TGR es establecida por los científicos (sociólogos) por convención, a la luz del problema (aplicación pretendida) a considerar. La convención no tiene porqué ser arbitraria, puede darse razón de ella en función del problema considerado.

En lo que concierne a $E$ ("evaluaciones"), que también es un concepto que constituye otro de los conjuntos base principales que establecen la ontología empírica de la teoría, su uso en la reconstrucción es algo peculiar una vez que el concepto sólo termina de ser aclarado cuando aparece en las funciones correspondientes. Sus entidades podrían ser, convencionalmente, tres cualesquiera, para esa reconstrucción se elige “+”, “-” y “?”. Pues bien, independiente de las especificidades de su uso en cada función, es evidente que sea cual sea la evaluación en cuestión (la positiva (+), la negativa $(-)$ y la neutra (?)) la determinación de cual sea el caso de una evaluación específica es independiente de TGR.

Claramente, todos los dominios base empíricos son TGR-no teóricos. Todos ellos pueden ser determinados de forma independiente de la teoría. Veremos ahora lo que pasa en el caso de las funciones y la relación que presenta TGR.

En lo que respeta a nor ("normativizar"), es la función que a un colectivo le asigna, en determinada circunstancia, cierta combinación de valores. Claramente esta función es determinada de modo independiente de TGR. La determinación de la combinación de valores que caracteriza un colectivo en determinada circunstancia puede darse a partir del comportamiento de los integrantes de los colectivos, cuando estos comportamientos manifiestan dichos valores. También puede darse por medio de la expresión del individuo (cuando conforma un colectivo unitario) o, por ejemplo, de líderes (para grupos). Puede determinarse el valor de la función por una investi- 
gación en documentos oficiales tales como los estatutos (grupos formales). Asimismo usando procedimientos técnicos más complejos. Por ejemplo, los valores asignados en determinadas circunstancias a las categorías sociales pueden ser determinados por encuestas, como, por ejemplo, aquéllas en las cuales los encuestados tienen que contestar ciertas cuestiones para saber si cada uno está habilitado a continuar haciendo la encuesta o no, y con ello determinar si forma parte o no de cierto colectivo; en caso de estar habilitado (esté dentro de los parámetros establecidos para la encuesta) se le pregunta acerca de lo que le resulta más importante, lo que le gusta, lo que no le gusta, y con ello se establecen la combinación de valores para ese individuo. La comparación del resultado de estas encuestas determina los valores de determinada categoría social.

También com, la función que asigna a un individuo, en determinada circunstancia, la aceptación activa, el rechazo activo o la indiferencia, dada la actitud del individuo con respecto a un conjunto (una combinación) de valores. Los valores que adquiera la función pueden ser determinados de modo independiente de la teoría una vez que se tengan establecidos los argumentos de la función. Dados un individuo y la circunstancia relevante, una vez determinada una combinación de valores, la observación de la actividad del individuo nos permite identificar cuál de las tres posibilidades $(+,-$, ?) es el caso.

En cuanto a asp ("aspirar"), la función que a un individuo, en una circunstancia, le atribuye una relación particular respecto de un colectivo, se recuerda que dicha relación puede ser de tres maneras: (i) de aspirar a pertenecer a este colectivo (+), (ii) de aspirar a no-pertenecer a este colectivo (-) o (iii) de ser indiferente con respecto a pertenecer a este colectivo (?). La función captura la actitud del individuo hacia el colectivo. Ésta también es una función que puede tener su valor determinado de modo independiente de TGR. Un método posible para dicha determinación es preguntarle al individuo directamente acerca de determinado colectivo si él aspira pertenecer, si aspira no pertenecer o si es indiferente en cuanto a eso. Otro modo de determinación sería la utilización de técnicas como la aplicación de cuestionarios o la realización de dinámicas de grupo. En ninguno de los tres casos es necesario presuponer la ley fundamental de TGR para que sea posible la determinación.

Finalmente tenemos gru ("ser grupo de referencia, para un individuo en una circunstancia"), una función que asigna a un individuo, en cierta circunstancia, un colectivo. Para que se pueda determinar qué colectivo debe ser asignado dado un individuo determinado y una circunstancia determi- 
nada, se requiere que, dado el cumplimiento de cierta condición, entonces se obtenga un resultado; la condición es la siguiente: que la aspiración del individuo en esa circunstancia con respecto a afiliarse a un colectivo tenga una evaluación $\beta$, es decir, que sea determinable la aspiración del individuo considerado, en cierta circunstancia, respecto a un determinado colectivo. El resultado debe ser que el comportamiento del individuo, con respecto a la normativa del colectivo en cuestión, tenga la evaluación $\beta$, es decir, el mismo valor que el obtenido para asp al aplicar esa función al individuo en cuestión, en la circunstancia considerada, respecto del mismo colectivo. Dicha relación entre la condición requerida y resultado esperado forma parte de lo que es la ley fundamental de TGR: la relación entre la condición, por un lado, y el resultado implicado, por otro lado, constituyen lo que expresa la ley fundamental de TGR que es ser un grupo de referencia. Única y exclusivamente de este modo es posible determinar el valor de gru. No hay otro modo de establecer si un colectivo es un grupo de referencia para un individuo en cierta circunstancia: no hay otra condición de prueba respecto de la que se pueda obtener un determinado resultado que permita establecer que un colectivo en determinada circunstancia es un grupo de referencia para un individuo. De este modo gru se caracteriza como una función TGRteórica, pues su determinación es dependiente de la TGR.

Sabiendo que apenas gru es el único miembro del conjunto de términos TGR-teóricos, ya se puede caracterizar a los modelos parciales de la teoría.

D3: $y=\langle I, C, V, S, E$, nor, com, asp $\rangle$ es un modelo parcial de la teoría de los grupos de referencia $\left(y \in \mathbf{M}_{\mathrm{pp}}(\mathrm{TGR})\right)$ syss existe un tal $x$ que:

(1) $x=\langle I, C, V, S, E$, nor, com, asp, gru $\rangle \in \mathbf{M}_{\mathrm{p}}$ (TGR)

(2) $y=\langle I, C, V, S, E$, nor, com, asp $\rangle$

\subsection{Condiciones de ligadura para TGR}

Acerca de condiciones de ligadura, en el caso de TGR, los conjuntos bases principales en cuestión son $I, C, V, S$ y $E$. A partir de estos conjuntos se dan (conjuntistamente) las funciones nor, com, asp y gru. Con la función nor se les asigna, en una circunstancia, un conjunto de valores a un colectivo. Parece plausible pensar que, bajo la misma circunstancia, un colectivo seguirá siempre com un mismo conjunto de valores como característico. Sin embardo, también parece plausible pensar que el cambio de circunstancia implica una variación, mismo que mínima, en el conjunto de valores característicos del colectivo. Del mismo modo, en el caso de com, es imposible afirmar que necesariamente el individuo se comportará del mismo modo en 
distintas circunstancias frente a un conjunto de valores característico de un colectivo. ${ }^{9}$ También en asp se debe notar que no es necesario que el individuo tenga la misma aspiración, en diferentes circunstancias, con respecto a un mismo colectivo. Por fin, tampoco parece ser necesario que un colectivo sea, independente de las circunstancias, un grupo de referencia para un individuo.

Sin embargo, en lo que se refiere a la función gru, hay cierta regularidad que parece ser interesante. ${ }^{10}$ Mismo no siendo necesario, parece ser constante para cierto indivíduo, mismo con cambio de circunstancia, que el mismo coletivo referencia para su conducta. ${ }^{11}$ Esto lleva a que se postule una condición de ligadura debilitada, es decir, en casi todos los casos suele suceder que:

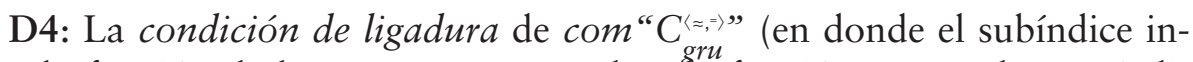
dica la función de la que se trata, a saber, la función gru, y el supraíndi-

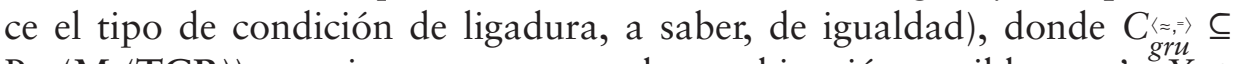
$\operatorname{Pot}\left(\mathbf{M}_{\mathrm{p}}(\mathrm{TGR})\right)$, requiere que, para toda combinación posible $x, x^{\prime} \in \stackrel{g r u}{X} \subseteq$ $\mathbf{M}_{\mathrm{p}}$ (TGR), se cumpla que en $X \subseteq \mathbf{M}_{\mathrm{p}}$ (TGR) se da la condición de ligaduragru sii para todo $x=\langle I, C, V, S, E$, nor, com, asp, gru $\rangle$, para todo $x^{\prime}=\left\langle I^{\prime}\right.$, $C^{\prime}, V^{\prime}, S^{\prime}, E^{\prime}$, nor', com', asp', gru' $\rangle$ y para todo $c, c^{\prime}, i, i^{\prime}, s, s^{\prime}:\langle c, i, s\rangle \in(C$ $\left.\frown C^{\prime}\right) \times\left(I \frown I^{\prime}\right) \times\left(S \frown S^{\prime}\right) \rightarrow\left(\operatorname{gru}(i, s)=\operatorname{gru}\left(i^{\prime}, s^{\prime}\right)\right)$.

9 Nótese que el comportamiento es relativo, además de a una circunstancia, no simplemente a un "conjunto de valores" sino a "un conjunto de valores característico de un colectivo" (recuérdese que en la tipificación de com se caracteriza el conjunto de valores en cuestión del siguiente modo: $\mathrm{D}_{\mathrm{II}}$ (nor), lo que significa que el comportamiento se refiere al conjunto de valores característico de un colectivo). Evidentemente que este "conjunto de valores" no tiene que ser idéntico en todas las aplicaciones de la teoría. El "contenido" del conjunto de valores aquí no tiene importancia, sino que sea "el conjunto de valores de (asociado a) un colectivo".

${ }^{10}$ Hay ciertas características que son constante em los indivíduos. Esa consistência (coerencia) constituyen su identidade. Esta es la perspectiva que embasa la postulación de esa condicción de ligadura debilitada.Además, situaciones como estas son muy estimulantes tanto para el sociólogo como para el filósofo de la ciencia. Queda el desafío de entender mejor, tanto desde la sociología como desde la filosofía de ciencia, la naturaleza de esta condición de ligadura debilitada específica y también la propia idea de condición de ligadura debilitada.

${ }^{11}$ Cabe notar que, por ejemplo, isso implica que el individuo siga con la misma aspiración en lo que respecta a filiarse colectivo. Eso también vale para el comportamiento del individuo. 


\subsection{Vinculos interteóricos para TGR}

Dado que una teoría no es una entidad aislada, capturar la identidad de una teoría requiere decir algo acerca de su entorno, es decir, las relaciones que mantiene dicha teoría con otras. Como se ha visto, este vínculo puede darse con conocimiento de sentido común, pre-teorías, proto-teorías, "folk-theories" o con teorías establecidas, es decir, con teorías cristalizadas (consensuadas) en la comunidad científica. En cualquiera de estos casos se trata de teorías presupuestas en el siguiente sentido: por medio de ellas se determinan los conceptos TRG-no-teóricos. Justamente por esto la relación gru no aparece, pues es un concepto TGR-teórico. También E, por ser un conjunto base auxiliar, no será llevado en consideración en lo que respecta a los vínculos interteóricos. Veamos qué se puede decir acerca de los demás conceptos presentes en la teoría.

Comencemos con I ("individuo"). Para determinar la extensión del concepto de individuo bastaría alguna "teoría" proveniente del conocimiento común; con esta "teoría" sería posible establecer la interpretación intencional básica de este conjunto base principal.

En lo que respecta a $C$ ("colectivo"), la determinación de la extensión del concepto de colectivo es un proceso básicamente "mecánico" en la medida que no se basa en una teoría (ya sea científica o de sentido común) sino que en ciertas reglas/características, a saber, las explicitadas en la caracterización de los modelos potenciales de TGR. Tenemos, entonces, este conjunto de reglas que, en cierto sentido, juega el papel de una teoría subyacente.

En el caso de $V$ ("valores"), tampoco existe una teoría de los valores a la cual Merton se refiera. Sin embargo, la noción de valor surge con la teorización ética/moral.

En el caso de $S$ (“circunstancias"), una noción aproblemática (en el sentido de que no se encuentra problematizada), proveniente del conocimiento común, es decir, la idea de sentido común de contexto.

En lo que concierne a $E$ ("evaluaciones"), también trata-se de una noción aproblemática, en el sentido recién mencionado. Del mismo modo, es posible decir que proveniente del conocimiento común.

En lo que respecta a nor ("normativizar"), nada en lo dicho por Merton hace referencia a una teoría de la atribución de valores. Asumamos que sea también una proto-teoría proveniente del conocimiento común lo que establezca, en cierto sentido, una atribución de valores en cierta circunstancia a determinado colectivo.

Con relación a com ("comportarse"), Merton también no se refiere a una teoría acerca de los comportamientos. Sin embargo, hay cierta concep- 
ción común de lo que es un comportamiento. Es, entonces, más un concepto que también proviene del conocimiento común.

En lo que respecta a asp ("aspirar"), del mismo modo que el concepto anterior, Merton no se refiere a ninguna teoría. Sin embargo, también en este caso, hay cierta concepción usual de lo que es aspirar/desear a algo. Como en el caso anterior, lo que se tiene es más un concepto que viene del conocimiento común.

El vínculo interteórico global de TGR (L(TGR)) es formado por la intersección de todos los vínculos interteóricos que tiene esta teoría con otros saberes subyacentes (presupuestos). Dado que todavía no hay disponibles reconstrucciones de las teorías subyacentes a TE no se presentará formalmente su vínculo teórico global.

\subsection{Las aplicaciones intencionales de TGR}

Algunos ejemplos de sistemas empíricos a que se pretende aplicar TGR, como hemos visto, son (Cf. Merton 1968, p. 323 [2002, p. 350]):

(a) El hecho de que sólo en un sentido estrecho y teóricamente superficial puede considerarse que el soldado raso del ejército que busca el ascenso siga una conducta diferente de la de un inmigrante que asimila los valores de un grupo nativo. En realidad son dos aplicaciones comparadas por su similitud estructural: por un lado, un individuo, en la circunstancia de ser soldado raso del ejército y buscar ascenso $[$ asp $(+)]$, según la propuesta de analogía entre las aplicaciones, asimila [com (+)] los valores de [nor] un colectivo superior en la jerarquía; por otro lado, un individuo, en la circunstancia de ser un inmigrante y asimilar $[$ com $(+)]$ los valores de [nor] un colectivo nativo, otra vez, según la propuesta de analogía, quiere $[a s p(+)]$ integrarse a este colectivo. En este ejemplo existen dos individuos distintos que presentan el mismo comportamiento referente a los valores de sus respectivos colectivos. La analogía entre las dos aplicaciones de la teoría puede considerarse el tipo de procedimiento que, según Kuhn (1962), ocurre cuando se intenta expandir las aplicaciones de una teoría teniendo por base algunas aplicaciones paradigmáticas, es decir, aprendiendo a ver cuáles son los aspectos relevantes de la semejanza entre las aplicaciones paradigmáticas.

(b) El hecho de que un muchacho en una zona de barrios bajos se oriente hacia los valores de un trabajador de casa de beneficencia y no hacia los valores de la pandilla de la esquina. En este caso se da una situación en la que se pueden observar dos comportamientos distintos: por 
un lado i), un individuo, en la circunstancia de ser un joven de una zona de barrios bajos y no orientarse a la pandilla de la esquina, se oriente hacia $[\mathrm{com}(+)]$ los valores de $[$ nor] un trabajador de casa de beneficencia (colectivo) y, por otro lado ii), este mismo individuo en la circunstancia de ser un joven de una zona de barrios bajos y orientarse hacia los valores de un trabajador de casa de beneficencia, no se oriente hacia [com (?)] los valores de [nor] la pandilla de la esquina (colectivo). Importa recordar que no orientarse a tales valores no es lo mismo que orientarse negativamente a dichos valores. A pesar de la vaguedad del ejemplo brindado por Merton, parece ser el caso que el individuo desea [asp] participar del colectivo de los trabajadores de casas de beneficencia y es indiferente [asp] con respeto a la pandilla de la esquina. El comportamiento con referencia a los valores de un colectivo es distinto del comportamiento con referencia a los valores de otro. Cada caso, estrictamente, es una aplicación diferente de TGR, aunque con una interdependencia entre ellas.

(c) El hecho de que un estudiante de Bennington abandone las ideas conservadoras de sus padres para adoptar las ideas más liberales de sus compañeros de colegio universitario. También en este caso se da una situación a partir de la cual se pueden observar dos comportamientos distintos: por un lado i), que un individuo en la circunstancia de ser estudiante de Bennington y de no adoptar hasta entonces ideas liberales, abandona [com (-)] las ideas conservadoras (valores) de [nor] sus padres (colectivo) y, por otro lado ii), este mismo individuo, en la circunstancia de ser estudiante de Bennington y de haber adoptado posiciones conservadoras en el pasado, adopta [com (+)] las ideas más liberales (valores) de [nor] sus compañeros de colegio universitario (colectivo). El comportamiento con referencia a los valores de un colectivo es distinto del comportamiento con referencia a los valores de otro. Nuevamente, cada caso, estrictamente, es una aplicación diferente de TGR, aunque con una interdependencia entre ella.

El hecho de que dos de los tres ejemplos (los ejemplos (b) y (c)) presenten una situación en la cual se describe dos comportamientos pudiera llevar a pensar que la aplicación de TGR requiere la comparación entre dos ellos. Aunque sea más intuitiva la presentación de los ejemplos de este modo, no cabe de hecho contemplar este tipo de comparación para la aplicación de la teoría; los dos comportamientos se conciben como aplicaciones autónomas de TGR. El ejemplo (a) muestra esto claramente. Lo más que cabe es, dado 
un comportamiento $x$ cualquiera que pudiera ser comparado con el propiamente considerado en la aplicación, es integrar $x$ como parte de parte de la circunstancia de dicha aplicación.

\section{Conclusiones}

En este artículo fue presentado un análisis de TGR. Como se ha dicho, se trata de una teoría que ha sido largamente utilizada en los más variados campos de las ciencias sociales con escasa consideración metateórica hasta ahora. Después de algunas aclaraciones preliminares, fue presentada una breve consideración informal de TGR. En secuencia, fue presentado el elemento teórico básico de la teoría. Este análisis de TGR demuestra ante todo que la Metateoría Estructuralista, mayormente conocida por sus aportes a las ciencias naturales, es también aplicable a las ciencias sociales. Estos pequeños pasos dados en esta dirección hacen plausible postular que, como ocurre en el caso de las ciencias naturales, la Metateoría Estructuralista puede contribuir significativamente a los debates llevados a cabo en la filosofía de la sociología. Especificamente, el análisis mostró que hay otros aspectos referentes ao comportamento de los indivíduos que están estrechamente vinculados a TGR. Asimismo, queda evidente que la noción de circunstancia amerita un estudio aprofundado, como también lo amerita la posibilidad de que el individuo tenga más de un colectivo como grupo de referencia en lo que atañe a una conducta. Finalmente, cada reconstrucción más que esté disponible constituyese en material concreto para discusión y/o comprobación de temas relacionados no sólo a la filosofia especial de la ciencia (especial = de una rama de saber dentro de una disciplina) sino de temas perninentes a la filosofia especial de la ciencia (en sentido mais amplo) y también de la filosofia general de la ciencia.

\section{Bibliografía}

Abreu, C. "La teoría de los grupos de referencia". Ágora - Papeles de Filosofía 31/2 (2012), 287-309. https://doi.org/10.15304/ag.31.2.1063

Abreu, C. "Análisis estructuralista de la teoría de la anomia". Metatheoria 4/2 (2014), 9-22.

Kuhn, T. The Structure of Scientific Revolutions. University of Chicago Press, Chicago, 1962. 
Lorenzano, P. y C. Abreu. "Las teorías de alcance intermedio de Robert K. Merton y las concepciones clásica y estructuralista de las teorías", pp. 482-492. En Martins, R. A., Lewowicz, L., Ferreira, J. M. H., Silva, C. C. \& L. A.-C. P. Martins (eds.). Filosofia e História da Ciência no Cone Sul. Seleção de Trabalhos do $6^{\circ}$ Encontro. Campinas: Associação de Filosofia e História da Ciência do Cone Sul (AFHIC), 2010.

Merton, R. K. Social Theory and Social Structure. Toward the Codification of Theory and Research. Illinois: The Free Press of Glencoe, 1949.

Merton, R. K. Social Theory and social Structure. 1968 enlarged edition. Illinois: The Free Press, 1968. (Versión castellana: Teoría y estructura sociales. México: Fondo de Cultura Económica, 2002).

Sota, E. La metáfora de "campo social": una lectura epistemológica de la sociología de Bourdieu. Villa María: Eduvim, 2013. 